\title{
TOLERÂNCIA DA SOJA AO MANGANÊS EM SOLUÇÃO NUTRITIVA EM TRÊS TEMPERATURAS ( $\left.{ }^{1}\right)$
}

\author{
HIPOLITO ASSUNÇĀO ANTONIO MASCARENHAS $\left({ }^{2,4}\right)$, CARLOS EDUARDO \\ DE OLIVEIRA CAMARGO $\left({ }^{3,4}\right)$, SONIA MARIA PIERRO FALIVENE $\left({ }^{2,4}\right)$ \\ e EDUARDO ANTONIO BULISANI $\left({ }^{2,4}\right)$
}

\begin{abstract}
RESUMO
Foram estudados dois cultivares de soja (Biloxi e Forrest) em soluções nutritivas contendo quatro níveis de manganês $(0,11 ; 2 ; 4$ e $6 \mathrm{mg} /$ litro) combinados com três diferentes temperaturas $\left(22,25\right.$ e $\left.28^{\circ} \mathrm{C}\right)$ em experimento efetuado em Campinas, em 1983. A tolerância foi medida, levando-se em consideração o peso seco das partes aéreas das plantas após 15 dias de cultivo nas soluções, os sintomas visuais de toxicidade e a concentração de manganês das folhas. Os resultados mostraram que, à medida que a temperatura das soluçōes variou de 28 para $22^{\circ} \mathrm{C}$, houve uma redução marcante no desenvolvimento das plantas dos dois cultivares, afetando, conseqüentemente, a prođução de matéria seca. A $25^{\circ} \mathrm{C}$ e ao nível de $2 \mathrm{mg} /$ litro de $\mathrm{Mn}$, foi possível distinguir o cultivar Forrest como sensível e Biloxi como tolerante, sendo esta combinação considerada ideal para fazer 'screening' de material genético. Esta separação, porém, poderia também ser feita nas temperaturas de 22 e $28^{\circ} \mathrm{C}$, respectivamente, nos niveis de 4 e $6 \mathrm{mg}$ /litro de $\mathrm{Mn}$. Na temperatura alta $\left(28^{\circ} \mathrm{C}\right.$ ), observou-se redução de toxicidade de $\mathbf{M n}$ para soja. O cultivar Biloxi apresentou, sob todas as temperaturas, as maiores concentrações de Mn na parte aérea.
\end{abstract}

Termos de indexação: nutrição mineral,Glycine max (L.) Merrill, melhoramento, temperatura, manganês, tolerância.

$\left.{ }^{1}\right)$ Trabalho parcialmente financiado por Transquímica Internacional Representaçōes Ltda., São Paulo, Companhia de Óleos Ve getais de Orlândia e Minasa S.A. Trabalho apresentado no XX Congresso Brasileiro de Ciêncià do Solo, em Belém, PA, de 14 a 21-7-1985. Recebido para publicação em 7 de março de 1984.

(2) Seção de Leguminosas, Instituto Agronômico (IAC), Caixa Postal 28, 13100 Campinas (SP).

$\left({ }^{3}\right)$ Seçāo de Arroz e Cereais de Inverno, IAC.

(4) Com bolsa de suplementação do CNPq. 


\section{INTRODUÇÃO}

Experimentos conduzidos sob as mais diferentes condições ambientes demonstraram existir variação na tolerância ao manganês tóxico do solo entre diferentes espécies de plantas (ANDREW \& HEGARTY, 1969, e MUNNS et alii, 1963) e entre cultivares dentro de uma mesma espécie (FOY et alii, 1969, e OUELLETTE \& DESSUREAUX, 1958). HEENAN \& CARTER (1977), utilizando $21-18$ e $33-28^{\circ} \mathrm{C}$ para temperaturas diurnas e noturnas respectivamente, e concentrações de 0,1 a $15 \mathrm{ppm}$ de $\mathrm{Mn}$, mostraram que a $15 \mathrm{ppm}$ e sob condições de temperatura mais elevada, houve maior desenvolvimento das plantas nos cultivares de soja Bragg e Lee, sem evidência de sintomas típicos de toxicidade de $\mathrm{Mn}$, sendo que o 'Bragg' se mostrou mais sensivel que 'Lee'. MASCARENHAS et alii (1981), em experimento de campo, verificaram que a $30^{\circ} \mathrm{C}$ não se evidenciaram os sintomas típicos de toxicidade de manganês nas folhas de soja; entretanto, a análise foliar mostrou teores elevados com redução na produção de grãos. Segundo MIRANDA et alii (1982), o "screening" de material genético em casa de vegetação, em solo com alto teor de $\mathrm{Mn}$, é possível somente durante o inverno, quando as temperaturas são mais amenas e os sintomas de toxicidade, mais evidentes.

O objetivo deste trabalho foi verificar o efeito de diferentes temperaturas sobre a tolerância de dois cultivares de soja em diferentes níveis de manganês, em solução nutritiva.

\section{MATERIAL E MÉTODOS}

O delineamento estatístico empregado foi de parcelas subdivididas, com duas repetições em distribuição inteiramente casualizada. As parcelas foram formadas por quatro concentrações de manganês $(0,11 ; 2 ; 4 \mathrm{e} 6 \mathrm{mg} / \mathrm{li}-$ tro) e as subparcelas, por dois cultivares de soja (Biloxi e Forrest), estudados em três temperaturas $\left(22,25\right.$ e $\left.28^{\circ} \mathrm{C}\right)$. Os cultivares foram escolhidos em virtude de Biloxi ter mostrado tolerância e Forrest, suscetibilidade, à toxicidade de Mn em trabalho anterior (MASCARENHAS et alii, 1984).

Sementes dos dois cultivares foram pré-germinadas durante 48 horas, selecionando-se vinte plântulas uniformes de cada um, as quais foram adaptadas em quatro telas de náilon: estas foram colocadas sobre quatro vasilhas plásticas de 8,3 litros de capacidade, contendo soluções nutritivas, de modo que as radículas ficassem em contacto com a solução e tivessem, portanto, um pronto suprimento de nutrientes.

A concentração das soluções nas quatro vasilhas plásticas foi a seguinte: $\mathrm{Ca}\left(\mathrm{NO}_{3}\right)_{2} \quad 0,4 \mathrm{mM}, \mathrm{MgSO}_{4} \quad 0,2 \mathrm{mM}, \mathrm{KNO}_{3} \quad 0,4 \mathrm{mM},\left(\mathrm{NH}_{4}\right)_{2} \mathrm{SO}_{4}$ $0,4 \mathrm{mM}, \mathrm{KH}_{2} \mathrm{PO}_{4} 0,05 \mathrm{mM}, \mathrm{H}_{3} \mathrm{BO}_{3} 1 \mu \mathrm{M}, \mathrm{CuSO}_{4} 0,03 \mu \mathrm{M}, \mathrm{ZnSO}_{4} 0,08 \mu \mathrm{M}$, 
$\mathrm{NaCl} 3 \mu \mathrm{M}, \mathrm{Na}_{2} \mathrm{MoO}_{4} \quad 0,01 \mu \mathrm{M}$ e $\mathrm{FeCl}_{3} 10 \mu \mathrm{M}$. A cada uma das quatro soluções foi adicionado $\mathrm{MnSO}_{4} .4 \mathrm{H}_{2} \mathrm{O}$, de modo a se obterem soluções com 0,$11 ; 2 ; 4$ e $6 \mathrm{mg} /$ litro de $\mathrm{Mn}^{2+}$. $\mathrm{O} \mathrm{pH}$ das soluções foi previamente ajustado a 4,8 com solução de $\mathrm{H}_{2} \mathrm{SO}_{4} 1 \mathrm{~N}$, sendo mantido constante por ajustamentos diários durante todo o experimento. As soluções foram continuadamente arejadas e as vasilhas plásticas contendo as soluções e plântulas, colocadas em banho-maria, com nivel de temperatura variando conforme o tratamerto. $\mathrm{O}$ experimento foi mantido com luz artificial em sua totalidade.

As plantas, após desenvolverem-se nessas condições por quinze dias, foram retiradas das soluções, separando-se as raízes e as partes aéreas das vinte plântulas de cada cultivar. As partes aéreas de cada tratamento foram colocadas para secar em estufa com temperatura de $60^{\circ} \mathrm{C}$ por cinco dias, quando foi determinado seu peso seco. As partes aéreas secas foram analisadas segundo método descrito por BATAGLIA et alii (1978) para obtenção dos teores de $\mathrm{P}, \mathrm{K}, \mathrm{Ca}, \mathrm{Mg}, \mathrm{Fe}, \mathrm{Mn}$ e $\mathrm{Zn}$. Foram calculadas equações de regressão do peso seco da parte aérea em função das concentrações de Mn na solução para as três temperaturas e para os dois cultivares estudados.

\section{RESULTADOS E DISCUSSÃO}

O efeito de temperatura foi marcante sobre o tipo e o grau de sintomas para os dois cultivares. Baixa temperatura, $22^{\circ} \mathrm{C}$, afetou muito mais o crescimento da planta do que o efeito tóxico de $\mathrm{Mn}$, ocasionando aparecimento de internódios bem curtos; foi observada clorose nas folhas apenas a partir de $4 \mathrm{mg} /$ litro de $\mathrm{Mn}$, para 'Biloxi' e 'Forrest', porém mais acentuada no último. Com a maturaçâo das folhas, a clorose ficou menos evidenciada. Os cotilédones foram bastante afetados, ficando mais amarelecidos e enrugados a $6 \mathrm{mg} /$ litro de $\mathrm{Mn}$ do que a $2 \mathrm{mg} /$ litro de $\mathrm{Mn}$, especialmente no 'Forrest'. Também, tanto ao nível de $4 \mathrm{mg} /$ litro como de $6 \mathrm{mg} / \mathrm{litro}$ de $\mathrm{Mn}$, observaram-se muitas pontuações castanhas nas folhas do 'Forrest', e somente a $6 \mathrm{mg} /$ litro em Biloxi. A $0,11 \mathrm{mg} /$ litro de $\mathrm{Mn}$, que foi a concentração testemunha, à temperatura de $22^{\circ} \mathrm{C}$, houve diminuição do tamanho das plantas porém não má-formação delas.

Embora as plantas tenham crescido normalmente, notou-se que em $2 \mathrm{mg} /$ litro foi possível separar o 'Forrest', que se mostrou mais suscetível que o 'Biloxi' através do enrugamento e clorose nas folhas. A $2 \mathrm{mg} /$ litro de $\mathrm{Mn}$, os cotilédones não foram afetados no 'Biloxi', mas no 'Forrest' foi observado amarelecimento e, à medida que as doses de $\mathrm{Mn}$ cresceram, houve maior efeito da toxicidade nos cotilédones em ambos. As plantas do 'Forrest' a 4 $\mathrm{mg} /$ litro de $\mathrm{Mn}$ mostraram enrugamento com clorose e muitas pontuações castanhas nas folhas, e a $6 \mathrm{mg} /$ litro de $\mathrm{Mn}$, por ocasião da colheita, as plantas estavam secas. A $28^{\circ} \mathrm{C}$, o 'Biloxi' também foi severamente afetado quan- 
do submetido às mais altas concentrações de $\mathrm{Mn}$ das plantas, com internódios bem mais compridos, enquanto na concentração de $2 \mathrm{mg} /$ litro de $\mathrm{Mn}$ não se evidenciou nenhum sintoma de toxicidade tanto nas folhas como nos cotilédones, para os dois cultivares. A $4 \mathrm{mg} /$ litro de $\mathrm{Mn}$, verificaram-se sintomas leves, como algum enrugamento nas folhas e amarelecimento dos cotilédones, enquanto, a $6 \mathrm{mg} /$ litro de $\mathrm{Mn}$, os sintomas foram mais acentuados, em particular no 'Forrest'.

No quadro 1 , pela análise estatística individual para cada temperatura, observa-se que houve significância ao nível de $1 \%$ para concentração de Mn (doses) e cultivares nas três temperaturas. A análise conjunta mostrou que a temperatura, concentração e cultivar foram importantes fatores, e a interação cultivar $x$ temperatura indicou que o comportamento do culti-

QUADRO 1 - Análise de variância para matéria seca da parte aérea de dois cultivares de soja, utilizando quatro níveis de $\mathrm{Mn}$ a 22,25 e $28^{\circ} \mathrm{C}$ em solução nutritiva e sua análise em conjunto




var apresentou variações sob diferentes temperaturas. As interações cultivar $\mathrm{x}$ doses (concentração de $\mathrm{Mn}$ ) e cultivar $\mathrm{x}$ temperatura $\mathrm{x}$ doses não foram significativas.

Pelo quadro 2, verifica-se que, à medida que a temperatura foi alterada de 28 para $22^{\circ} \mathrm{C}$, houve uma redução marcante no desenvolvimento da planta, afetando, consequentemente, a produção de matéria seca. A $25^{\circ} \mathrm{C}$, os dois cultivares de soja se diferenciam bastante um do outro, sendo que $o$ valor seletivo do 'Biloxi' é $82 \%$ e, do 'Forrest', 68\%. Já para as temperaturas de 22 e $28^{\circ} \mathrm{C}$ esta mesma tendência é observada para 4,0 e $6,0 \mathrm{mg} / \mathrm{litro}$ de manganês.

QUADRO 2 - Produção média de matéria seca da parte aérea de dois cul tivares estudados em solução nutritiva com níveis de 0,$11 ; 2 ; 4$ e $6 \mathrm{mg} /$ litro de $\mathrm{Mn}$ e às temperaturas de 22,25 e $28^{\circ} \mathrm{C}$

\begin{tabular}{|c|c|c|c|c|c|c|c|c|c|c|c|c|}
\hline \multirow{4}{*}{$\begin{array}{l}\text { Tratamento } \\
\text { mg/litro de Mn }\end{array}$} & \multicolumn{12}{|c|}{ Matéria seca da parte aérea } \\
\hline & \multicolumn{4}{|c|}{$22^{\circ} \mathrm{C}$} & \multicolumn{4}{|c|}{$25^{\circ} \mathrm{C}$} & \multicolumn{4}{|c|}{$28^{\circ} \mathrm{C}$} \\
\hline & \multicolumn{2}{|c|}{ 'Biloxi } & \multicolumn{2}{|c|}{ 'Forrest' } & \multicolumn{2}{|c|}{ 'Biloxi } & \multicolumn{2}{|c|}{ 'Forrest' } & \multicolumn{2}{|c|}{ 'Biloxi' } & \multicolumn{2}{|c|}{ 'Forrest' } \\
\hline & $\mathrm{mg}$ & $\%$ & $\mathrm{mg}$ & $\%$ & $\mathrm{mg}$ & $\%$ & $\mathrm{mg}$ & $\%$ & $\mathrm{mg}$ & $\%$ & $\mathrm{mg}$ & $\%$ \\
\hline 0,11 & 110 & 100 & 100 & 100 & 136 & 100 & 118 & 100 & 186 & 100 & 136 & 100 \\
\hline 2 & 100 & 91 & 80 & 80 & 111 & 82 & 80 & 68 & 167 & 90 & 126 & 93 \\
\hline 4 & 90 & 82 & 60 & 60 & 97 & 71 & 68 & 58 & 157 & 88 & 118 & 87 \\
\hline 6 & 70 & 80 & 60 & 60 & 84 & 62 & 62 & 51 & 155 & 83 & 88 & 65 \\
\hline
\end{tabular}

Os resultados de produção de matéria seca dos dois cultivares mostram que altas temperaturas reduzem o efeito de toxicidade de Mn em soja. Aparentemente a diminuição de toxicidade de $\mathbf{M n}$ sob alta temperatura $\left(28^{\circ} \mathrm{C}\right)$ não foi devida à redução de concentração de $\mathrm{Mn}$ na parte aérea, uma vez que houve aumento dessa concentração à medida que se aumentou a temperatura em ambos os cultivares (Quädro 3), confirmando dados de HEENAN \& CARTTER (1977) observados para soja e outras culturas (MUNNS et alii, 1963, e BOLOSKO \& SMITH, 1971). Embora na análise de variância para matéria seca (Quadro 1) a interação tripla (cultivar $\times$ temperatura $\mathrm{x}$ doses de $\mathrm{Mn}$ ) não tenha sido estatisticamente significativa, os presentes resultados mostraram comportamento diferencial de cultivares em relação à toxicidade de $\mathrm{Mn}$, avaliada pela concentração do micronutriente nas folhas, principalmente sob temperaturas baixas $\left(22 \mathrm{e} 25^{\circ} \mathrm{C}\right)$. O 'Biloxi' apresentou sempre maior concentração de Mn na parte aérea em relação ao 'For- 
rest' (Quadro 3); pode-se assumir que as diferenças entre cultivares para tolerância à toxicidade em temperaturas mais baixas $\left(22\right.$ e $\left.25^{\circ} \mathrm{C}\right)$ não foram devidas, de per si, à concentração das soluções, tendo em vista que em ambos ela atingiu valores elevados, não ocorrendo, portanto, para cultivar tolerante, mecanismo de exclusão. Com exceção do manganês, os teores de macro e micronutrientes na parte aérea, tanto para o cultivar Biloxi como para o Forrest, não apresentaram variações evidentes à medida que se aumentaram os níveis de Mn nas soluções.

Pela figura 1 , verifica-se maior inclinação da reta da equação de regressão entre matéria seca da parte aérea e concentração do manganês na solução a $25^{\circ} \mathrm{C}$ em relação às retas correspondentes às temperaturas de $22 \mathrm{e}$ $28^{\circ} \mathrm{C}$; portanto, as diferenças em matéria seca a $25^{\circ} \mathrm{C}$ são maiores para o mesmo aumento na concentração de $\mathrm{Mn}$ na solução do que nas outras temperaturas. Os dados apresentados no quadro 2 e na figura 1 permitem considerar que $25^{\circ} \mathrm{C}$ seria a temperatura mais adequada para avaliação da toxicidade de manganês em soja.

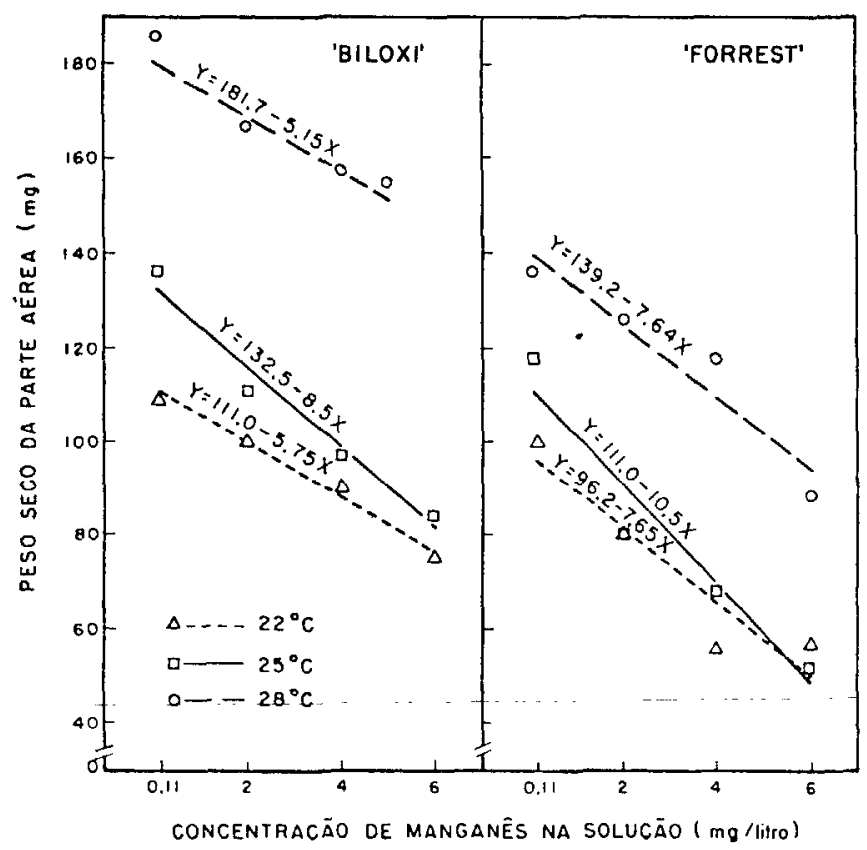

FIGURA 1 - Relação entre o peso seco da parte aérea e a concentração de manganês na solução nutritiva a três temperaturas na raiz para dois cultivares de soja. 


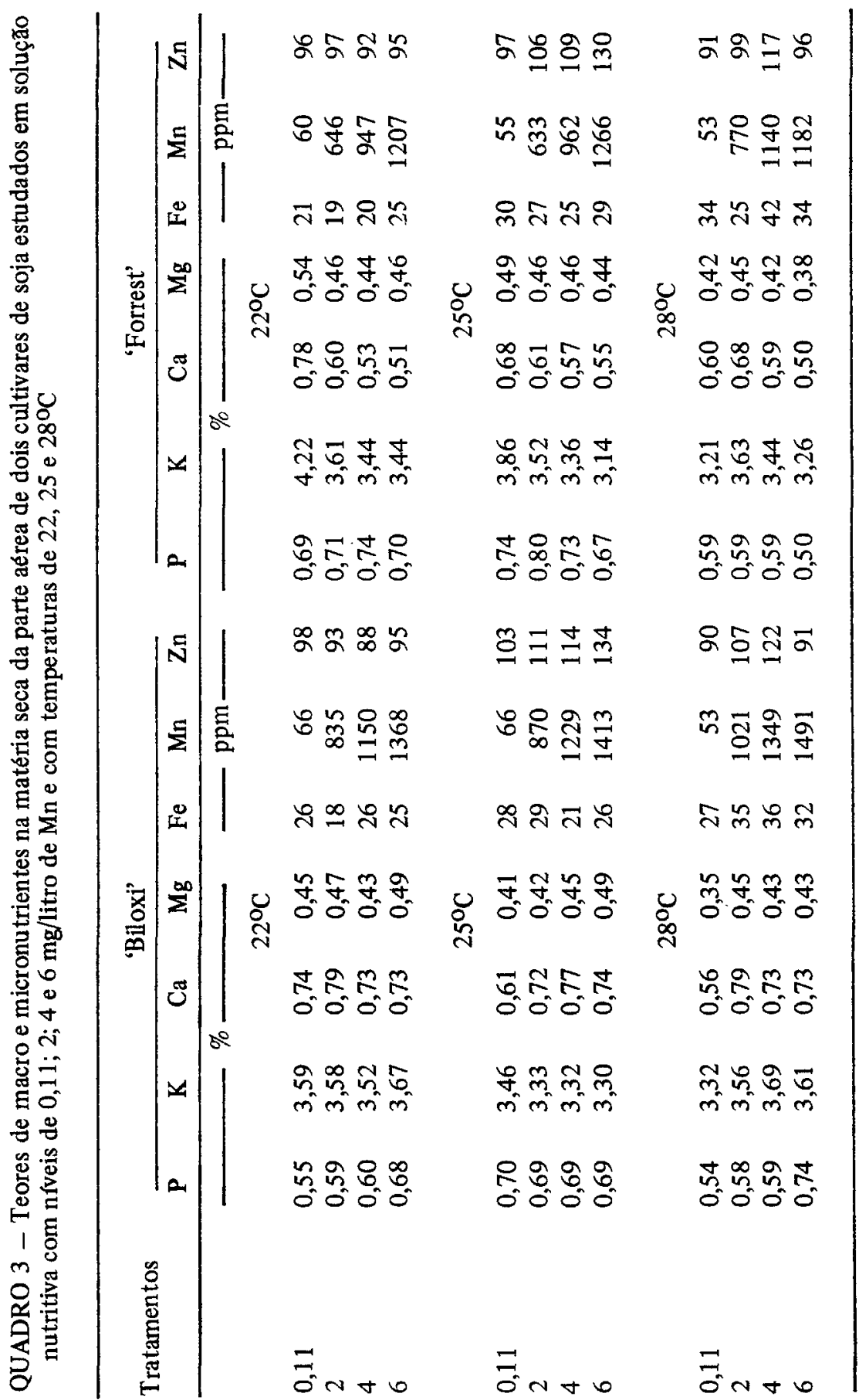




\title{
SUMMARY \\ SOYBEAN TOLERANCE TO MANGANESE IN NUTRIENT SOLUTION UNDER THREE TEMPERATURES
}

\begin{abstract}
Two scybean cultivars (Biloxi and Forrest) were grown under three different temperatures $\left(22,25\right.$ and $\left.28^{\circ} \mathrm{C}\right)$ in nutrient solutions containing four levels of manganese $(0.11 ; 2 ; 4$ and $6 \mathrm{mg} / 1)$ in an experiment carried out at Campinas, in 1983. Mn tolerance differences were evaluated by dry matter weight of the above ground parts after a period of 15 days of growth. The results showed that as the temperature were reduced from 28 to $22^{\circ} \mathrm{C}$ there was a reduction in the plant growth of the two cultivars which affected the dry matter weight. At $25^{\circ} \mathrm{C}$ and at the level of $2 \mathrm{mg} / 1 \mathrm{of}$ Mn, Forrest was suceptible and Biloxi, tolerant, being this condition appropriate for screening soybean germoplasm for $\mathrm{Mn}$ tolerance. This separation could also be done at 22 and $28^{\circ} \mathrm{C}$ at 4 and $6 \mathrm{mg} / 1$ levels of $\mathrm{Mn}$, respectively. High temperature $\left(28^{\circ} \mathrm{C}\right)$ showed reduction of $\mathrm{Mn}$ toxicity in both soybean cultivars. The cultivar Biloxi always showed at all temperatures greater concentration of Mn than Forrest.
\end{abstract}

Index terms: mineral nutrition, Glycine max (L.) Merrill, breeding, temperature, manganese, tolerance.

\section{AGRADECIMENTOS}

Os autores agradecem aos funcionários Ângela Maria Caldeira da Silva e Francisco Vidal Filho, da Seção de Leguminosas, a colaboração prestada, e à Seção de Química Analítica a análise química de material vegetal.

\section{REFERENNCIAS BIBLIOGRÁFICAS}

ANDREW, C.S. \& HEGARTY, M.P. Comparative response do manganese excess of eight tropical and four temperate pasture legumes. Australian Journal of Agricultural Research, 20(4):687-696, 1969.

BATAGLIA, O.C.; TEIXEIRA, J.P.F.; FURLANI, P.R.; FURLANI, A.M.C. \& GALLO, J.R. Métodos de análise química de plantas. Campinas, Instituto Agronômico, 1978. 31p. (Circular, 87)

BOLOSKO, J.A. \& SMITH, D. Influence of temperature and nitrogen fertilization on growth and composition of switch grass (Panicum vergatum L.) and timothy (Phleum pratensi L.) at anthesis. Agronomy Journal, 63: 853-857, 1971 .

FOY, C.D.; FLEMING, A.L. \& ARMINGER, W.H. Differential tolerance of cotton varieties to excess manganese. Agronomy Journal, 61:690-693, 1969. 
HEENAN, D.P. \& CARTER, O.G. Influence of temperature on the expression of manganese toxicity by two soybean varieties. Plant and Soil, 47:219-227, 1977.

MASCARENHAS, H.A.A; BRAGA. N.R.; BULISANI, E.A.; FEITOSA, C.T.; HIROCE, R. \& BATAGLIA, O.C. Efeito do corretivo sobre soja cultivada em solo de cerrado contendo $\mathrm{Al}$ e $\mathrm{Mn}$. In: SEMINÁRIO DE PESQUISA DE SOJA, 2., Brasília, 1981. Anais. v.2, p. 567-573.

; CAMARGO, C.E.O. \& FALIVENE, S.M.P. Comportamento de cultivares de soja em soluçao nutritiva contendo diferentes nfveis de manganês. Bragantia, Campinas, 43(1):201-209, 1984.

MIRANDA, M.A.C. de; MASCARENHAS, H.A.A.; BULISANI, E.A.; VALADARES, J.M.S. \& HIROCE, R. Comportamento de dois cultivares de soja em função de Mn do solo. Bragantia, Campinas, 41: 135-143, 1982.

MUNNS, D.M.; JOHNSON, C.M. \& JACOBSON, L. Uptake and distribution of manganese in oat plants. I. Varietal variation. Plant and Soil, 19:115$125,1963$.

OUELLETTE, G.I. \& DESSUREAUX, L. Chemical composition of alfalfa as related to degree of tolerance to manganese and aluminum. Canadian Journal of Soil Science, 38:206-214, 1958. 